\title{
High Power Conversion Efficiencies and Scaling Issues for Multimode Vertical-Cavity Top-Surface-Emitting Lasers
}

\author{
K. L. Lear, Member, IEEE, S. P. Kilcoyne, and S. A. Chalmers
}

\begin{abstract}
We report advances in the power conversion efficiencies of vertical-cavity top-surface-emitting lasers defined by proton implantation. Efficiencies as high as $13.4 \%$ and $15.8 \%$ have been obtained for single-mode and multimode operation, respectively. Scaling issues are addressed including the size dependence of threshold current, series resistance, lasing output power, and power conversion efficiency. We find that devices between $15 \mu \mathrm{m}$ and $25 \mu \mathrm{m}$ diameters show the highest power conversion efficiency due to the threshold current not scaling with the conductance and output power. Device geometries with contact apertures both equal to and less than (overlapping) the active region diameter were investigated.
\end{abstract}

$\mathbf{V}$ ERTICAL-cavity surface-emitting lasers (VCSELs) present a large parameter space for epitaxial designs including the number, location, composition, and thickness of quantum wells in the active region; the composition, thickness, and doping of cladding layers; and the composition, grading, doping, and number of distributed Bragg reflectors (mirrors). Additionally, various active region areas can be defined by implantation or etching. Depending upon application specific requirements, optimum designs are evaluated with regard to appropriate metrics such as threshold current density, threshold voltage, maximum output power density, slope efficiency, series resistance, and power dissipation. All of these parameters impact the electrical to optical power conversion efficiency so that it may be used as a global performance metric prior to the definition of application specific requirements.

VCSEL power conversion efficiencies have increased substantially during the past two years. Peters et al. have achieved a maximum continuous-wave $(\mathrm{CW})$, room-temperature power conversion efficiency of $17.3 \%$ in a bottom-emitting, multimode, index-guided device [1]. We have previously reported on proton-implanted, top-emitting VCSELs that exhibited a power conversion efficiency of $12.7 \%$ during single-mode operation [2]. In this letter we describe the extension of our earlier work to larger devices in which the maximum $\mathrm{CW}$, room-temperature power conversion efficiency occurs during multimode operation with a value of $15.8 \%$. In addition to modal characteristics, increases in the active region diameter

Manuscript received March 8, 1994; revised April 22, 1994. This work was supported by the United States Department of Energy under Contract DE-AC04-94AL85000.

The authors are with Sandia National Laboratories, Photonics Research Department, Albuquerque, NM 87185 USA.

S. A. Chalmers is currently at P. O. Box 4517, Berkeley, CA 94704 USA. IEEE Log Number 9402763. affect the electrical, thermal, and other optical properties of the laser. The associated parameters scale differently with device size resulting in changes in performance metrics. These scaling issues are discussed with respect to their impact on power conversion efficiency.

The epitaxial structure and device processing used in this study were similar to ones described in detail in [2]. The molecular beam epitaxial layers were grown on an n-type GaAs substrate and included a 33 period n-type AlGaAs mirror, a triple InGaAs quantum well in a one-wave cavity of graded AlGaAs, and a 22 period p-type AlGaAs mirror. The mirror alloy composition was graded as described previously [3], and both the silicon and beryllium doping concentrations were decreased near the active region to reduce free carrier absorption [4], [2]. The nominal design wavelength was $980 \mathrm{~nm}$. Two differences from the structure and processing described in [2] are that a delta-doped GaAs cap was not used and the contact metal was alloyed at $350^{\circ} \mathrm{C}$ prior to the implant. The process employed a relatively thin $(2000 \AA) \mathrm{AuBe}$ contact metallization to permit ion implantation through the contact. After initial measurements, the finished devices were then annealed at $400^{\circ} \mathrm{C}$ to reduce implant damage near the contacts and near the junction. This temperature was found to optimize laser efficiency [5].

A variety of device sizes were fabricated on the sample with photoresist implant mask nominal diameters of 10,15 , 25 , and $35 \mu \mathrm{m}$. This dimension $g$ defines the gain or active region and will be referred to as such in this letter. In general the diameter of the aperture in the metallic contact $a$ was the same as the implant mask ( $a=g$ ), but devices with $g=15 \mu \mathrm{m}$ and $a=10 \mu \mathrm{m}$ as well as $g=35 \mu \mathrm{m}$ and $a=25 \mu \mathrm{m}$ were also fabricated. In these special cases where $a<g$, the metal contact overlaps into unimplanted regions to provide lower contact and lateral spreading resistances; however this is at the expense of output power since part of the gain region is obscured. The cross-section of the completed structure is shown in Fig. 1.

The devices were characterized in wafer form on a probe station. Room-temperature CW light versus current and voltage versus current measurements were made as previously described [2]. The lasing wavelength varied with radial position on the wafer, so measurements were made at a position where the threshold current of small devices was minimum. The lasing wavelength in this area was approximately $970 \mathrm{~nm}$. Five randomly selected devices of each size described above 


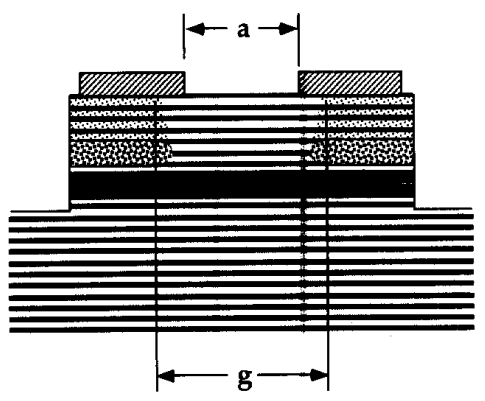

Fig. 1. Schematic drawing of the cross-sectional structure of a proton-implant defined VCSEL electrically isolated by etching. The contact aperture $a$ and the nominal implant mask diameter which defines the active region size $g$ were varied. For most devices $a=g$, although some devices with $a<g$ were also evaluated. These latter devices showed reduced threshold currents, series resistances, output powers, and efficiencies.
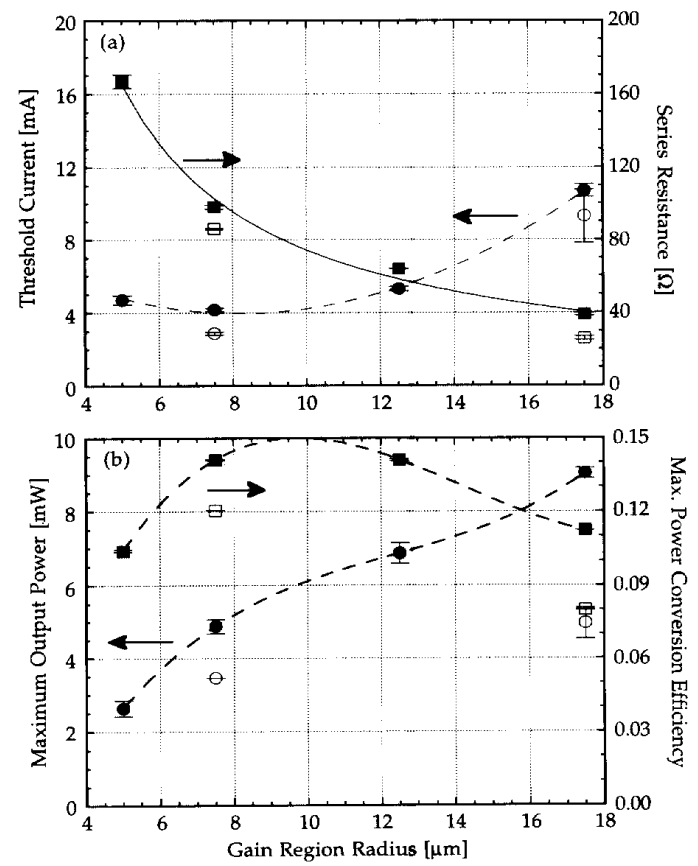

Fig. 2. (a) The average threshold currents (circles) and series resistances (squares) and (b) the maximum power (circles) and maximum power conversion efficiencies (squares) for VCSELs as a function of the active region radius $(r=g / 2)$ for VCSELs as a function of the active region radius $(r=g / 2)$. The solid symbols indicate devices with equal aperture and active region diameters $(a=g)$ while the open symbols indicate devices with a reduced aperture $(a<g)$. The solid line indicates the fit of $R_{s}=A / r+B /\left(\pi r^{2}\right)$ to the resistance data for devices with $a=g$. The dashed lines are polynomial curves intended only as aids to the eye. Error bars indicate the standard deviation of the data.

were measured and the average statistics for these devices are shown in Fig. 2.

Fig. 2(a) shows two parameters, threshold current and series resistance, as a function of device radius. These parameters affect power conversion efficiency, and it is useful to analyze their dependence on device size. Ideally, in the absence of edge effects and current crowding, the threshold current density should be constant giving a threshold current that is proportional to the active region area. The threshold current density was calculated as the ratio of threshold current to active area even though the current density varies radially within the active region [6], [7]. The two largest VCSELs, 25 and 35 $\mu \mathrm{m}$ in diameter, have approximately equal threshold current densities of $1 \mathrm{kA} / \mathrm{cm}^{2}$. However, the smaller devices have much higher threshold current densities. This is partly due to effects that require additional currents which scale with the device circumference, such as nonradiative recombination that occurs at the periphery of the implant. Interestingly, the data in Fig. 2(a) shows not only an increase in threshold current density for smaller devices, but even an increase in the threshold current for the smallest (10 $\mu \mathrm{m}$ diameter) device. Peripheral currents alone cannot account for this behavior; rather there must be increased optical losses (diffraction and unabsorbed spontaneous emission) associated with the smallest device. It is also noteworthy that the lasers employing a contact aperture smaller than the gain region $(a<g)$ have smaller thresholds than devices with the same gain region and contact aperture diameter $(a=g)$ as previously observed by others [8]. Metal overlying a portion of the active region may reduce the optical loss and thus decrease the threshold current.

The device resistance as plotted in Fig. 2(a) was taken from a linear fit of the current versus voltage curves between the lasing threshold and maximum output power points on the curves. The current versus voltage relationship is quite linear in this range and extrapolates to approximately $1.4 \mathrm{~V}$. Decreasing the aperture diameter to be less than the gain region diameter $(a<g)$ reduces the resistance slightly in comparison to the devices with $a=g$. The reduction comes from reduced lateral current flow and lower contact resistance [2], [8]. The data for resistance of the four device sizes with $a=g$ have been fit with the expression $R_{s}=A / r+B /\left(\pi r^{2}\right)$ for gain region radius $r=g / 2$ where the first term accounts for constriction or spreading, lateral, and contact resistances that scale inversely with radius and where the second term corresponds to a uniform vertical current flow resistance that scales inversely with the gain area. The best fit is obtained with coefficient values of $A+0.066 \Omega$-cm and $B=2.6 \times 10^{-5} \Omega$ $\mathrm{cm}^{2}$. Using these coefficients, the two terms would be equal for $r=1.3 \mu \mathrm{m}$; thus the $1 / r$ term dominates for all the device sizes in the present study. This highlights the importance of enhancing lateral as well as vertical conductivity in VCSEL mirror stacks.

Fig. 2(b) shows two performance metrics, maximum power and maximum power conversion efficiency, as functions of the device size. While the maximum output power of the lasers increases with gain region size, it does not increase as rapidly as the gain region area so that the effective maximum power intensity decreases with size. In fact, the maximum power approaches a linear relationship in radius for the larger devices. This is a result of current crowding that concentrates the carriers and thus power at the periphery of the device under high level injection conditions [6], [7].

Since the junction impedance is much higher under the lower level injection conditions near threshold, the current injection is more uniform and the threshold current is approximately proportional to the area of large devices as noted 
above. Thus, while the conductance and power vary linearly in radius for moderate to high currents, the threshold current varies sublinearly with radius $\left(\propto r^{n}\right.$ with $n<1$ ) for small devices due to periphery effects and super-linearly with radius $(n>1)$ for large devices. The net results is an optimization of the relative threshold current, and correspondingly power conversion efficiency, at intermediate sizes. This is the behavior seen in Fig. 2(b) for the maximum power conversion efficiency. A smooth interpolation predicts that a gain diameter between 15 and $25 \mu \mathrm{m}$ would give the highest value for this epitaxial design. Different designs are likely to have similar trends although the optimum values and sizes will depend on the structure and fabrication.

Further incremental improvements in device efficiency, primarily through a decrease in threshold current, were observed after several hours of laser operation [2], [5], [9]. The lasers were annealed at $400^{\circ} \mathrm{C}$ for 30 seconds prior to testing and burn in. Both furnace and current annealing are believed to reduce nonradiative recombination traps created by ion implant damage near the active region [2], [5], [10]. Fig. 3(a) shows the characteristics for a VCSEL with a $15 \mu \mathrm{m}$ diameter gain region and contact aperture. The device had been current annealed at $12 \mathrm{~mA}$ for 40 hours with the power increasing from 4.35 to $4.48 \mathrm{~mW}$. The lasing threshold is $3.9 \mathrm{~mA}$ and the maximum power before thermal rollover is $5.5 \mathrm{~mW}$. The pronounced kink in the output power curve at $7.5 \mathrm{~mA}$ coincides with another transverse mode beginning to lase in addition to the fundamental mode. The maximum single-mode power is $2.0 \mathrm{~mW}$. The voltage curve shows a nearly constant resistance of $97 \Omega$ at currents above the lasing threshold. The curve's linearity indicates that thermionic barriers at the mirror heterointerfaces do not significantly contribute to the total device resistance. The extrapolation of the linear portion of the curve to zero current yields $1.4 \mathrm{~V}$, the expected idealized turn-on voltage corresponding to a GaAs bandgap energy. This confirms that there are no other significant nonohmic contributions to the voltage drop other than the p-n junction voltage.

The power conversion efficiency for this device is plotted in Fig. 3(b). There are multiple local maxima that correspond to different collections of transverse modes lasing. The first maximum in power conversion efficiency has a value of $13.4 \%$ and occurs at $6.6 \mathrm{~mA}$ where the laser is operating single-mode. The global maximum in efficiency is $15.8 \%$ and occurs at 10.2 $\mathrm{mA}$ where the laser is operating in a few transverse modes. We believe that this is the highest reported power conversion efficiency for top-emitting VCSELs and that $13.4 \%$ is the highest reported single-mode power conversion efficiency for any VCSEL structure.

In conclusion, we have reported advances in the power conversion efficiencies of top-emitting VCSELs defined by proton implant. Values as high as $13.4 \%$ and $15.8 \%$ have been obtained in single-mode and multimode operation of a 15 $\mu \mathrm{m}$ diameter laser operating $\mathrm{CW}$ at room temperature. Scaling issues and their effect on power conversion efficiencies have been examined. These lasers show resistance and power scaling that are indicative of current crowding at the periphery of the device. This occurs because contact, lateral, and substrate

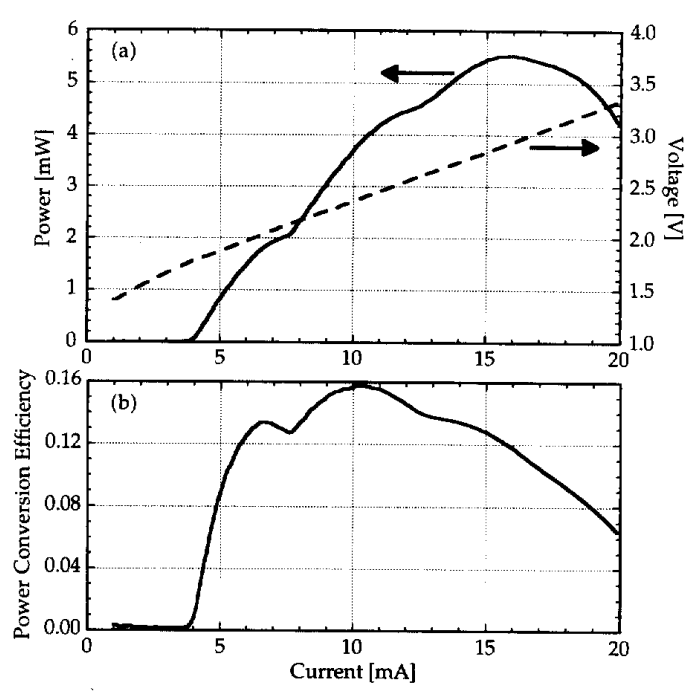

Fig. 3. The $\mathrm{CW}$, room-temperature characteristics of a $15 \mu \mathrm{m}$ diameter VCSEL after current annealing subsequent to the $400^{\circ} \mathrm{C}, 30$ second rapid thermal anneal. The curves, as functions of the drive current are (a) output power (solid), device voltage (dashed), and (b) power conversion efficiency.

spreading resistance contributions are greater than the vertical mirror resistance. The lasers also show an increase in threshold current for small devices indicating increased optical losses and constant threshold current densities for the largest devices. The disparity between the scaling of the threshold current and the effective device area results in optimum efficiencies for intermediate sized devices. Larger devices would benefit from better current injection uniformity, through reduced lateral resistance and perhaps transparent conducting contacts such as indium-tin-oxide or cadmium-tin-oxide [11], and from improved heat-sinking.

\section{ACKNOWLEDGMENT}

The authors would like to thank J. Nevers and J. Escobedo for technical assistance, and R. Hadley and J. Hohimer for useful discussions.

\section{REFERENCES}

[1] M. G. Peters, D. B. Young, F. H. Peters, J. W. Scott, B. J. Thibeault, and L. A. Coldren, " $17.3 \%$ peak wall plug efficiency vertical-cavity surface-emitting lasers using lower barrier mirrors," Photon. Technol. Lett., vol. 6, pp. 31-33, 1994.

[2] K. L. Lear and S. A. Chalmers, "High single-mode power conversion efficiency vertical-cavity top-surface-emitting lasers," Photon. Technol. Lett., vol. 5, pp. 972-974, 1993.

[3] S. A. Chalmers, K. L. Lear, and K. P. Killeen, "Low resistance wavelength-reproducible p-type $(\mathrm{Al}, \mathrm{Ga}) \mathrm{As}$ distributed Bragg reflectors grown by molecular beam epitaxy," Appl. Phys. Lett., vol. 62, pp. 1585-1587, 1993.

[4] G. Hasnain, K. Tai, N. K. Dutta, Y. H. Wang, J. D. Wynn, B. E. Weir, and A. Y. Cho, "High temperature and high frequency performance of gainguided surface emitting lasers," Electron. Lett., vol. 27, pp. 915-916, 1991.

[5] K. L. Lear, S. A. Chalmers, and S. P. Kilcoyne, "Effects of implant annealing on the power conversion efficiency of vertical cavity topsurface emitting lasers," in Proc. IEEE Lasers and Electro-Optics Soc. 1993 Annu. Meeting, San Jose, CA, 1993, pp. 546-547. 
[6] J. W. Scott, R. S. Geels, S. W. Corzine, and L. A. Coldren, "Modelling temperature effects and spatial hole burning to optimize vertical-cavity surface-emitting laser performance," IEEE J. Quantum. Electron., vol. $29,1295-1307,1993$.

[7] R. Michalzik and K. J. Ebeling, "Modeling and design of protonimplanted ultralow-threshold vertical-cavity laser diodes," IEEE $J$. Quantum Electron., vol. 29, pp. 1963-1974, 1993.

[8] R. A. Morgan, G. D. Guth, M. W. Focht, M. T. Asom, K. Kojima, L. E. Rogers, and S. E. Callis, "Transverse mode control of verticalcavity top-surface-emitting lasers," Photon. Technol. Lett., vol. 4, pp. 374-377, 1993.
[9] D. Vakhshoori, J. D. Wynn, R. E. Leibenguth, and R. A. Novotny, "Long lasting vertical-cavity surface-emitting lasers," Electron. Lett., vol. 29, pp. 2118-2119, 1993.

[10] P. Ressel, H. Strunsny, S. Gramlich, U. Zeimer, J. Sebastian, and K. Vogel, "Optimised proton implantation step for vertical-cavity surfaceemitting lasers," Electron. Lett., vol. 29, pp. 918-919, 1993.

[11] L.-W. Tu, E. F. Schubert, H. M. O'Bryan, Y.-H. Wang, B. E. Weir, G. J. Zydzik, and A. Y. Cho, "Transparent conductive metal-oxide contacts in vertical-injection top-emitting quantum well lasers," Appl. Phys. Lett., vol. 58, pp. 790-792, 1991. 